Pecvnia, 4 (2007), pp. 27-47

\title{
Un paradigma de la nueva economía: el sector financiero en España
}

\author{
José Luis Fanjul Suárez \\ jlfans@unileon.es \\ Laura Valdunciel \\ laura.valdunciel@unileon.es \\ Universidad de León \\ Dpto. de Dirección y Economía de la Empresa \\ Fac. de Ciencias Económicas y Empresariales \\ Campus de Vegazana, s/n \\ 24071 León (España)
}

En los modelos de estrategia empresarial convencionales, las empresas decidían las tecnologías a emplear, en cambio, actualmente, es la tecnología la que dirige las decisiones estratégicas en la empresa asumiendo un rol desconocido hasta ahora.

La forma de gestionar los negocios, ha modificado los supuestos económicos fundamentales sobre los que se sustentaban la mayoría de las empresas, transformando la economía industrial en lo que hoy llamamos economía digital. Este nuevo paradigma ha variado la configuración de las estrategias empresariales, tal y como, se concebían antes de la aparición de este nuevo fenómeno.

El impacto de esta "nueva economía" por sectores está siendo desigual, el sector financiero ha sido pionero en el uso de Internet como nuevo 
canal de distribución. El desarrollo de Internet permitirá conseguir un crecimiento sostenido debido a que la distribución de productos financieros no requiere un intercambio físico de bienes, ya que el dinero es desde hace ya mucho tiempo, un bien electrónico.

En este trabajo se pretende hacer un breve análisis de la situación de la banca online en España, de las estrategias adoptadas por las entidades financieras más importantes de nuestro país, y de cómo ha influido este nuevo canal en los productos financieros que ofrecen estas entidades.

Palabras clave: TIC, Nueva Economía, Banca Online. new distribution channel. Internet development allows a sustainable growth because the distribution of financial products does not require a physical exchange of goods. The reason is that money has been, for a long time, an electronic good.

This paper tries to analyze the situation of online banking in Spain, the strategies adopted by the most important financial concerns in the country, and how this new channel has influenced the financial products offered by those concerns.

Key words: CIT, New Economy, Online Banking.

\section{CONTEXTO MACROECONÓMICO DE LA NUEVA ECONOMÍA}

Durante los años noventa, la economía norteamericana vivió uno de los momentos más importantes. La nota diferencial respecto de los ciclos económicos precedentes radicaba, en este caso, en dos elementos de una naturaleza claramente diferente pero, al mismo tiempo, muy relacionados: el factor tecnológico y la instauración definitiva del proceso de globalización.

En la teoría económica tradicional, el componente tecnológico ha formado parte de las diferentes teorías sobre el crecimiento y desarrollo económicos. Los economistas diseñan sus recetas de política económica guiados por unos patrones de comportamiento que, en base a la experiencia previa, claramente suelen mostrar las relaciones entre los problemas económicos y los objetivos a largo plazo perseguidos.

El contexto macroeconómico en el que se desencadenó la nueva economía se caracterizó por la simultaneidad de un elevado ritmo de crecimiento del PIB, una fuerte reducción del desempleo y una moderación de las tasas de inflación. No se sabía con certeza cuál podía ser el detonante de lo que algunos economistas reconocieron como una "stagflación"1 de signo contrario, ni qué factores podían haber llevado a una situación en la que el crecimiento de la producción y el empleo no generaban tensiones inflacionistas. La ruptura de esos patrones por parte

Stagflación, conocido como estanflación o situación macroeconómica en la que se dan bajas tasas de crecimiento el PIB con inflación y desempleo. 
de la economía de EEUU abrió las puertas a la definición de un nuevo concepto: la nueva economía².

El factor tecnológico, nunca desdeñado en los modelos tradicionales del crecimiento, cobró gran importancia en este instante, en la medida que provocó una revolución productiva sin precedentes que trascendía el mercado nacional y se propagaba en el mercado mundial. Los modelos económicos del comercio internacional habían incorporado en su formulación la importancia del factor tecnológico como determinante de la ventaja comercial. Implícitamente en unos casos y explícitamente en otros, estos modelos convencionales no entendían el progreso técnico como se concibe tras la incorporación de las TIC. La diferencia de esta revolución tecnológica respecto de las precedentes es que no se limita a desplazar la función de producción de los sectores afectados, sino que desplaza la frontera de posibilidades de innovación haciendo que su capacidad transformadora sea muy elevada al igual que su rentabilidad. El análisis más profundo de la estructura productiva reveló que el aumento de productividad registrado en la economía americana estaba respondiendo a los efectos de un factor innovador y, más concretamente, de una tecnología muy específica: la de la información y las comunicaciones. La emergencia de un nuevo sector, alentado por las posibilidades de ganancia a corto plazo en los mercados financieros, fue rápidamente absorbida por el mercado. Desde un punto de vista estrictamente económico, las TIC han producido una amplia gama de efectos que han modificado las pautas de evolución de variables macroeconómicas y microeconómicas. La nueva economía ha favorecido el paso de un sistema basado en los servicios y métodos tradicionales a otra menos material, menos tangible y más innovador que despliega sus efectos a diferentes niveles. Por un lado y como hemos señalado con anterioridad, es evidente que el desarrollo y la aplicación de las tecnologías de la información y la comunicación tienen una influencia en la evolución de macromagnitudes como el PIB.

La gráfica adjunta refleja la tasa de crecimiento del PIB (Figura 1) para tres de las economías más importantes a precios constantes, es decir con valores deflactados, y permite comprobar que la economía norteamericana mantiene una clara diferencia respecto de las otras dos.

\footnotetext{
2 De hecho aunque comúnmente el término "nueva economía" está muy establecido en nuestros días, los expertos aún discuten sobre su verdadero significado y contenido.
} 
En cuanto a los efectos sobre la contención de los precios, parece claro que las empresas tienen una oportunidad para reducir sus costes a través del incremento de la productividad total de sus factores. Sin embargo, parece lógico preguntarse si las TIC han sido la verdadera causa de la fase expansiva del ciclo económico norteamericano. Los estudios en este sentido son amplios y parecen corroborar los argumentos iniciales. Morrow y Roëguer (2001), muestran que el progreso tecnológico ha sido determinante en el crecimiento de la economía americana. Según sus trabajos, la participación del trabajo en el PIB ha disminuido al igual que la acumulación de capital en favor de un progresivo aumento del factor tecnológico. El resultado es un incremento del PIB potencial en más de un punto por encima de las estimaciones para la UE-15.

Sin embargo, esa expansión de la producción posiblemente no habría sido posible sin una evolución favorable de los precios de esas nuevas tecnologías. La consideración de los precios hedónicos ${ }^{3}$ ha facilitado el conocimiento de la influencia que la reducción real de precios ha tenido en el proceso de sustitución de otros equipos y mano de obra por los bienes de capital de la nueva economía.

No obstante este tipo de bienes no sólo admiten consideraciones de naturaleza macroeconómica también se rodean de aspectos de naturaleza microeconómica. Argandoña (2001), señala entre estos rasgos los que citamos a continuación. Se trata de "bienes de experiencia", es decir, es necesario que se conozcan para que puedan ser valorados. El hecho de conocerlos no provoca una pérdida de valor, sino más bien puede promover el efecto contrario. Se trata de bienes que suelen generar economías de escala debido a la existencia de altos costes fijos hundidos de entrada, costes marginales muy bajos o nulos y ausencia de restricciones de capacidad. Las condiciones de no rivalidad y no exclusión ${ }^{4}$; aparecen como una clara nota diferencial de los bienes no públicos que complementa las dos anteriores. Por último se señalan como rasgos adicionales el que se trata de bienes no espaciales y que permiten los efectos en red lo que favorece la aparición de externalidades.

\footnotetext{
Precios hedónicos: descomposición del precio total en la satisfacción de unas necesidades del consumidor a través de las características que incorpora cada modelo de producto.

4 En este sentido hay que tener en cuenta que, normalmente los bienes que reúnen estas características son los bienes públicos, bienes normalmente producidos por el Gobierno.
} 
Figura 1. Tasa de crecimiento del PIB

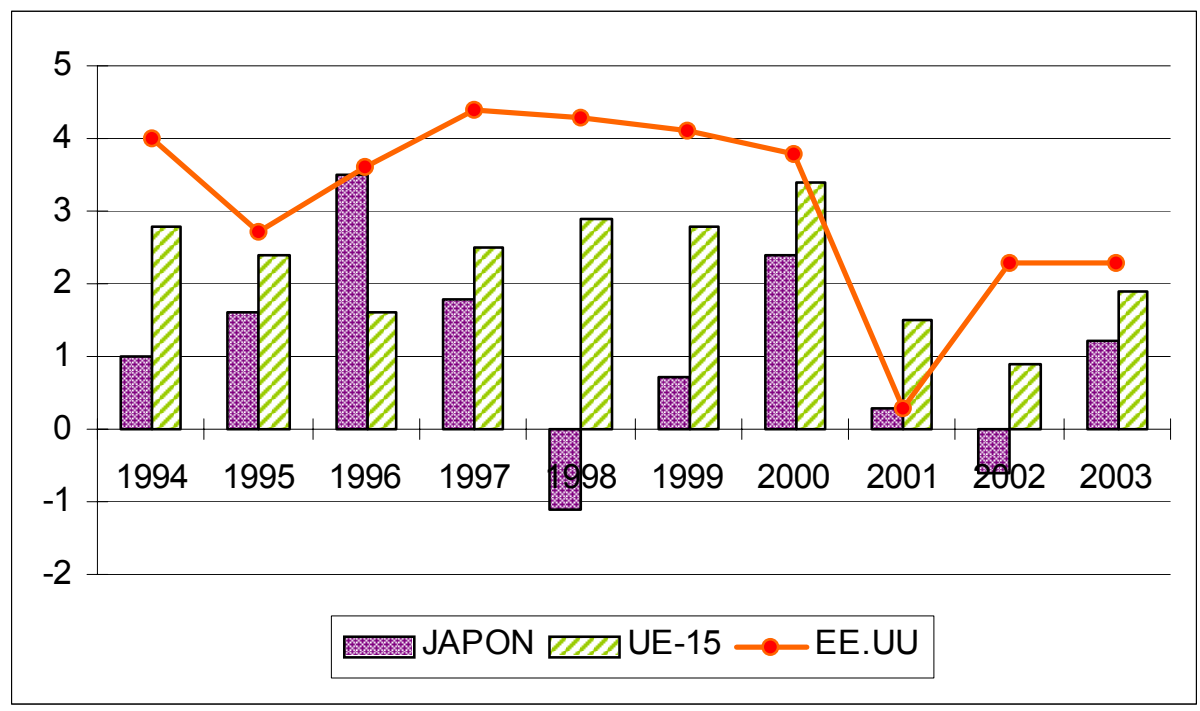

Fuente: N. González (2003).

\section{EL IMPACTO DE LA NUEVA ECONOMÍA}

En el apartado anterior hemos mostrado la influencia que las TIC han tenido en los principales agregados macroeconómicos, sin embargo, y a pesar de que estos efectos han sido importantes, es a nivel microeconómico donde con más efectividad han desplegado sus efectos. Las empresas han sido los agentes económicos que con más rapidez, han absorbido el factor tecnológico en sus funciones de producción, mejorando con ello su eficiencia, eficacia y en definitiva el resultado final de sus ejercicios. Es por ello por lo que resulta de especial interés analizar de que manera, la incorporación a nivel microeconómico de las TIC ha podido variar la concepción tradicional del mercado. La forma de gestionar los negocios, ha modificado los supuestos económicos fundamentales sobre los que se sustentaban la mayoría de las empresas, transformando la economía industrial en lo que hoy llamamos economía digital. Este nuevo paradigma ha variado la configuración de las estrategias empresariales, tal y como, se concebían antes de la aparición de este nuevo fenómeno. En los modelos de estrategia empresarial convencionales, las empresas decidían las tecnologías a emplear, en cambio, el nuevo modelo implica, que es la tecnología la que dirige las decisiones estratégicas en la empresa asumiendo un rol desconocido hasta ahora. 
El efecto revolucionario de Internet implica algo más que un cambio en el entorno competitivo de las empresas tradicionales y que la entrada de competidores nuevos y dinámicos. Internet está modificando no sólo la forma en que actuamos y vivimos, sino también el modo de expresarnos. Este nuevo fenómeno ha abierto las puertas a la aparición de nuevos conceptos lingüísticos y económicos, como por ejemplo e-banco, e-conomía, e-comercio, etc. (Andersen Consulting 2000).

A pesar de que estamos inmersos en el momento actual en una nueva "era tecnológica", es sabido que una vez que el mercado absorbe e incorpora a sus procesos de decisión el impacto de este nuevo cambio, la tendencia normal del ciclo económico es retornar a su equilibrio natural y buscar un nuevo objetivo que alcanzar a largo plazo. Si hace algunos años la informática era un elemento diferenciador que colocó a la empresa en la vanguardia tecnológica, actualmente esto ya no es un factor diferencial, puesto que se considera que este avance ha sido ya asimilado por el entorno empresarial. Así, la novedad que ha supuesto Internet y la economía digital, en poco tiempo desaparecerá asumiéndose como algo habitual. Según Craig Barrett, consejero delegado de Intel: "En cinco años no habrá compañías de Internet porque todas las compañías serán de Internet... o no existirán". No obstante, no todos los países ni todos los mercados han mostrado un mismo grado de permeabilidad hacia esta economía digital.

Tomemos como ejemplo la Unión Europea. En el año 2003, la Unión Europea cuenta con aproximadamente 200.000.000 usuarios de Internet, un $116,7 \%$ más que en el año 2000 , lo que representa un $43,5 \%$ de la población total del espacio comunitario. Los países del norte de Europa: Suecia (76,8\%), Holanda (66\%), Dinamarca (62,5\%), y Reino Unido $(60,6 \%)$ cuentan con una mayor tasa de utilización de Internet entre sus habitantes, en contraposición con los países del sur de la Comunidad como es el caso de España (34,5\%), Chipre (22\%), Portugal $(19,2 \%)$ y Grecia $(15,3 \%)$.

Las cifras anteriores, dibujan un mapa que pone de manifiesto la brecha tecnológica existente entre el norte y sur de Europa, y permite intuir una potencial correlación entre el crecimiento económico y la penetración de las TIC. Si tomamos como referencia lo que ocurre en el sector empresarial español, nos encontramos con un importante volumen de empresas que aceptan las reglas del juego que impone el nuevo mercado electrónico, pero que al mismo tiempo, rehúsan incorporar los cambios necesarios en su estructura, auto limitándose ellas mismas el acceso a 
este nuevo mercado. Centrándonos en el comercio electrónico, la Asociación Española de Comercio Electrónico (AECE-FECEMD) estima las ventas de empresas a consumidores finales B2C (Business to Consumer), correspondientes al año 2003, en 1.530 millones de euros (Figura 2), y en un $23 \%$ de los internautas ${ }^{5}$, aquellos que realizan compras a través de Internet, siendo casi un $43 \%$ de los españoles usuarios habituales de la red (Figura 3).

Figura 2. Cifra de ventas de comercio electrónico a consumidores finales (B2C)

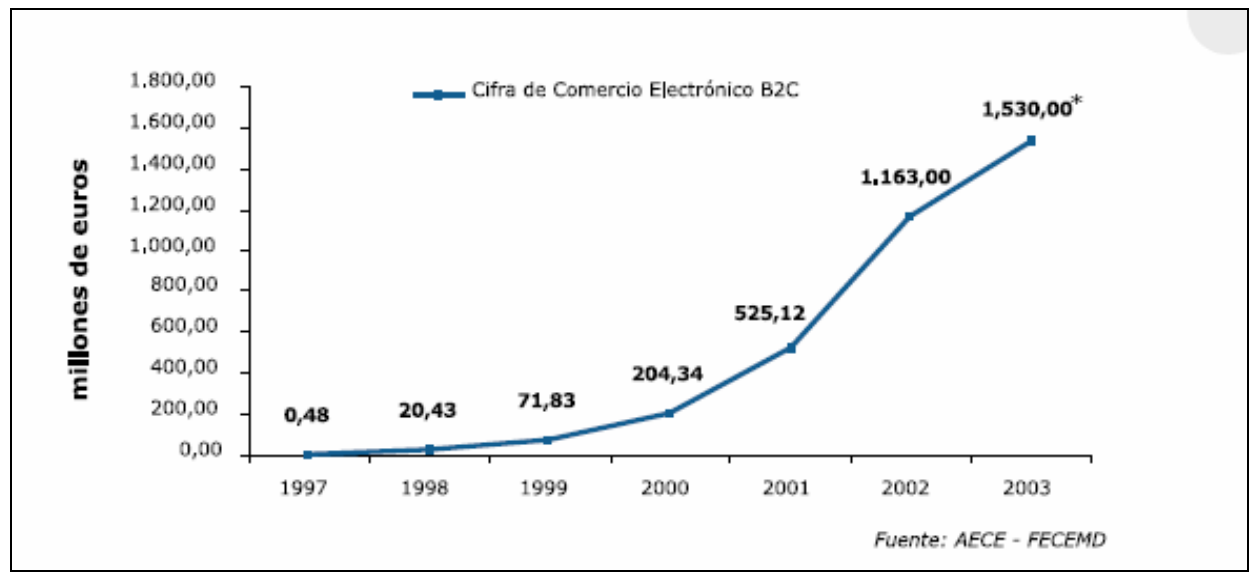

Figura 3. Cifra de internautas y de compradores españoles a través de Internet ${ }^{6}$

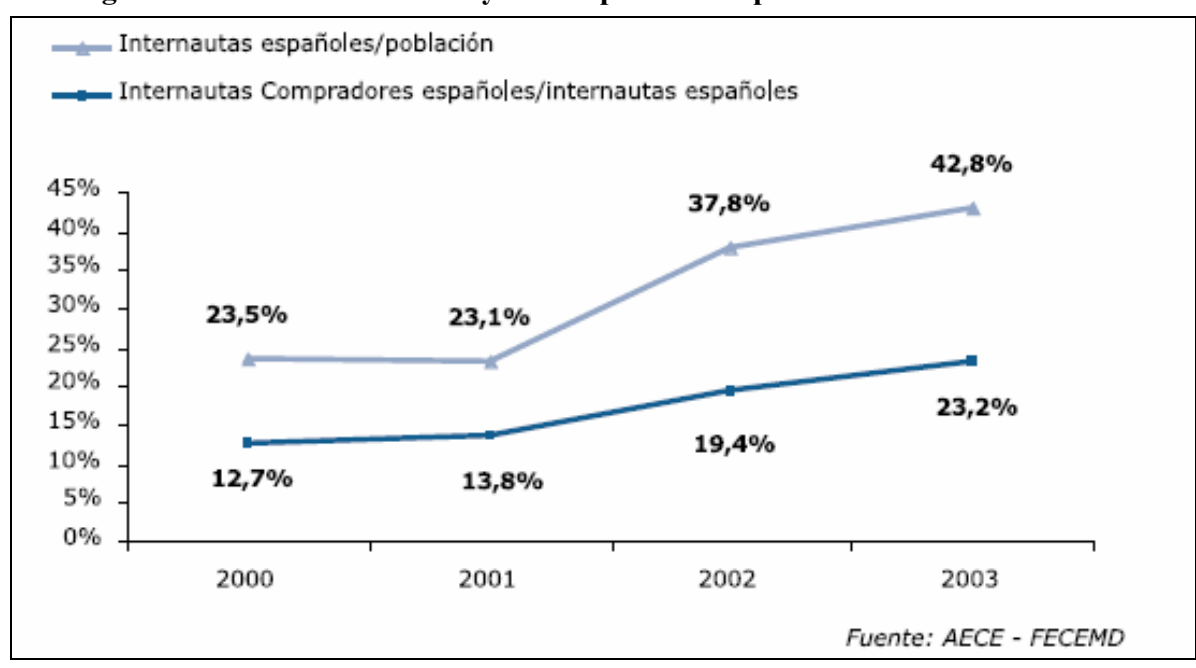

$5 \quad$ Aquellos españoles que se conectan a Internet más de una vez al mes.

6 Los datos de la gráfica son extraídos de los estudios de AECE-FECEMD y hacen referencia al mes de marzo de cada año. 


\section{LA ECONOMÍA DIGITAL}

Para analizar la "Nueva Economía" tenemos que ver cuáles son los rasgos comunes de todas las definiciones, las que aportan los autores escépticos y las de los autores convencidos de su existencia. El principal rasgo común en toda la literatura es considerar la "Nueva Economía" como una revolución tecnológica.

Amit y Zott (2001), encuentran la esencia de la denominada Economía Digital en una extraordinaria presencia de efectos o Economías de Red, entendiendo por Economías de Red aquellas en las que el valor de un producto o servicio para un usuario depende no sólo del producto en sí mismo, sino del número de usuarios que utilicen o consuman ese producto. Esta peculiaridad ha sido la nota dominante en otros sectores a lo largo de la historia, por lo que no podemos considerarla exclusiva de la era de la información. Sin embargo, se trata de un principio fundamental en el entorno digital, desde el momento en que todo lo que se inscribe en él encuentra su origen y una parte sustancial de su explicación en el término Economías de Red.

La incertidumbre que ha envuelto a la Economía Digital en sus comienzos ha provocado que algunos autores hayan replanteado ciertos principios económicos considerados como básicos e irrefutables en épocas precedentes. Sin embargo, cuando analizamos estudios sobre la "Nueva Economía", descubrimos que dichos estudios se apoyan en los mismos paradigmas, los mismos conceptos y las mismas teorías que los estudios de la economía tradicional. La ley de la oferta y la demanda sigue siendo aplicable también en los negocios que operan a través de la Red, la cadena de valor de Porter (Porter 2001) sigue siendo también un marco válido para analizar los procesos de creación de valor de las empresas "punto com" y la técnica de flujos libres de caja de tesorería sigue siendo una de las más utilizadas a la hora de realizar la valoración de empresas en la "Nueva Economía".

Los paradigmas, los conceptos y las teorías que se utilizan en el estudio de la "Nueva Economía" aparentemente son los mismos que los que se utilizan en la economía tradicional pero con nuevas aplicaciones, matizaciones y desarrollos del cuerpo teórico tradicional.

Porter y Millar (1985) reconocen, ya a mediados de los ochenta, el importante papel que juegan las tecnologías de la información en el proceso de creación de valor a partir de las aportaciones iniciales de Porter en 1980. Hess y Kemerer (1994) validaron el supuesto de reducción 
de los costes de transacción en los mercados electrónicos. Gurbaxani y Whang (1991) estudian las fronteras de los mercados electrónicos teniendo en cuenta los costes de transacción y los costes de agencia. Bakos (1991) y Bakos y Brynjolfsson (1993) estudian tanto las razones por las que en los mercados electrónicos las empresas negocian con un número limitado de proveedores, y modelizan el comportamiento de los consumidores a partir de la reducción de los costes de búsqueda en los mercados electrónicos.

A finales de los años noventa se empiezan a estudiar los aspectos microeconómicos de una red de comunicación, de manera que Internet comienza a ser considerada la infraestructura de numerosos mercados electrónicos. Así, Bakos (1998) discute los efectos de Internet sobre los mercados electrónicos. Brynjolfsson y Smith (2000) comparan los mercados para productos vendidos tanto por canales tradicionales como a través de Internet.

Desde el punto de vista macroeconómico, la investigación ha seguido dos vertientes: Una primera línea de investigación se inició a principios de los años noventa, intentando resolver el problema conocido como la paradoja de la productividad de las tecnologías de la información (Solow 1987; Berndt y Morrison 1995; Brynjolfsson y Yang 1996). Este problema consistía en el hecho de que a pesar de que las empresas americanas realizaban importantes inversiones en tecnologías, contrariamente a lo planteado por los académicos, la productividad del factor trabajo no aumentaba. Es a finales de los años noventa (Jorgenson y Stiroh 1995; Brynjolfsoon y Hitt 1996; Greenan y Mairesse 1996; McAfee 2000a) cuando se empieza a obtener evidencia empírica de que este incremento de la productividad se está produciendo y por tanto, de que las inversiones en tecnologías de la información están siendo rentables.

Una segunda línea de investigación, se centra en el impacto de la Economía Digital sobre el crecimiento, el empleo, la productividad y la inflación (Haltiwanger y Jarmin 2000; Greenstein 2000).

\section{CARACTERÍSTICAS DE LA ECONOMÍA DIGITAL}

Las bases económicas que diferencian y caracterizan a la nueva economía se resumen en las siguientes: 


\section{Desintegración vertical}

En la economía tradicional, a las empresas no les resulta muy fácil poseer muchos de los eslabones de la cadena de valor, lo cual implica altos costes de interacción y transformación que provocan altos niveles de integración vertical. En cambio con la economía digital, se pueden alcanzar niveles de calidad, flexibilidad y ahorro mediante acuerdos con socios tecnológicamente punteros e innovadores, especializados en determinados eslabones de la cadena de valor.

No obstante, la mayoría de las empresas ya establecidas poseen gran parte de su cadena de valor y las nuevas empresas, cuyos modelos estén basados en la economía digital, deben desarrollarse continuamente manteniendo modelos flexibles, graduales, y que incorporen nuevos tipos de operaciones antes de integrarse verticalmente para mejorar la calidad, la velocidad o el servicio al cliente.

\section{Beneficios sobre activos intangibles}

En la economía tradicional, la rentabilidad del mercado depende en gran parte de los activos físicos. Así la propiedad, las fábricas y los equipos tienen un gran peso específico en la mayoría de los balances de las empresas. Los activos intangibles, como pueden ser la marca, I+D y las relaciones con los clientes, aunque aportan un valor considerable en sí mismos, no son considerados una fuente de ingresos.

En cambio en la economía digital, a medida que los mercados van siendo segmentados los activos intangibles se valoran por encima de los físicos. Al no depender ya del conjunto de activos físicos, los activos intangibles, pueden ser desarrollados fácilmente a bajo coste a través de una base global de clientes, convirtiéndose así en una fuente independiente de ingresos y de valor.

Teniendo en cuenta esto, las nuevas empresas deben impulsar continuamente el valor de la propiedad intelectual y de las relaciones con los clientes, verdadero núcleo del negocio, con el fin de incrementar el volumen y los márgenes, mientras resisten la tentación de adquirir activos físicos para reducir costes a corto plazo. 


\section{Rendimientos crecientes}

En la economía industrial, los rendimientos decrecientes de las inversiones en plantas y equipo industrial implican que maximizar los beneficios significa limitar la capacidad de la producción.

La ley de los rendimientos decrecientes significa que hay espacio para la competencia en la mayoría de los sectores económicos. Esta regla se puede aplicar también en la economía digital, sólo en aquellos negocios que basan su input principal en activos físicos, pues la información, las relaciones con los clientes y la propiedad intelectual, no están limitadas por la capacidad de la fábrica. De hecho, una empresa puede crecer sin límite aproximando los costes unitarios a cero y aumentando exponencialmente el valor del producto para el cliente.

En este contexto, las nuevas empresas deben redefinir continuamente las ofertas que realizan a sus clientes con la finalidad de reducir sus costes, evitando competir exclusivamente en precio.

El acceso a la información ha dejado de ser caro y restringido

En la economía tradicional, la información para los compradores y vendedores es a menudo cara y difícil de conseguir. Los fabricantes y minoristas llevan a cabo investigaciones de mercado para conocer las necesidades y comportamientos de los consumidores, con dificultades para segmentarlas.

En la economía digital, la aparición de canales de distribución de gran alcance y eficiencia hace que la información sea más barata y fácil de obtener. Los clientes son cada vez más exigentes debido a la gran cantidad de ofertas y al fácil acceso a una información más completa, lo que hace que el consumidor tenga cada vez mayor poder de compra.

Esta ventaja que le ofrece al cliente la economía digital también puede serlo para el vendedor, pues éste puede recopilar información sobre sus clientes y utilizarla para poder ofrecer a cada uno de ellos exactamente lo que desea facilitándoles productos y servicios de forma personalizada. 
Ya no se necesitan varios años ni grandes capitales para establecer un negocio a escala mundial

En la economía industrial abrir nuevos mercados requiere un gran esfuerzo económico y tiempo para realizar estudios de mercado, construir activos físicos, identificar y elaborar inventarios específicos, y generar la capacidad necesaria de producción y venta. En la actualidad, se pueden abrir nuevos mercados en plazos mucho más cortos. Las redes de valor pueden utilizarse para buscar proveedores de productos y servicios, para promocionar, vender y dar soporte a los mismos de forma más rápida y eficiente que en la economía tradicional.

Las empresas de nueva creación se introducen rápidamente en los mercados logrando una apreciable cuota de negocio (se estima que los brokers online en EE.UU. se han apoderado ya del 30\% del mercado entre particulares) debiendo, no obstante continuar innovando sus ofertas para alcanzar diferentes mercados, y reforzar sus marcas comerciales.

En un estudio realizado por Andesen Consulting (2000), se han recogido las respuestas adoptadas por las empresas españolas ante la economía digital entre las cuales enumeramos las más frecuentes:

- "Esperar y ver" cómo funciona Internet: El 26\% de las empresas españolas entrevistadas no tenían presencia en Internet pues no lo consideraban una necesidad real y creían que el comercio electrónico no aportaba valor añadido a su oferta.

Creación de una página web con contenido informativo: Esta respuesta tampoco aporta mucho ya que el uso que se ha hecho de los canales de comunicación electrónicos hasta ahora, consiste principalmente en la promoción en Internet y en la comunicación interna por medio de una Intranet, con escaso impacto sobre el negocio.

Creación de una página web con contenido transaccional: Casi la mitad de las empresas entrevistadas consideraban que la venta de productos a través de este canal no era aplicable a corto plazo. Un $16 \%$ de los encuestados ya utilizaban Internet para vender sus productos o servicios y solo el $10 \%$ de las grandes empresas afirmaron que no venderían sus productos a través de este canal en los próximos dos años. Sin embargo la mayoría de las empresas no creían que este canal fuera a ser importante en el volumen de las operaciones de su negocio (un 29\% de las empresas pensaba que no llegará al $1 \%$ de sus operaciones). 
- Reorientación del negocio: A pesar de que la mayoría de las empresas opinaron que el comercio electrónico afectaba a la estructura y gestión de las mismas, sigue considerándose como una cuestión tecnológica de la que se ocupa, normalmente, el departamento de informática.

El $89 \%$ de las empresas encuestadas opinó que en su empresa no se aprovechaban adecuadamente todas las posibilidades del comercio electrónico, señalando ésta como una de las principales barreras al desarrollo del mismo en nuestro país.

\section{UN CASO DE ESTUDIO: EL SECTOR FINANCIERO EN ESPAÑA}

El sector financiero ha incorporado la tecnología de la información con gran rapidez por ser intensivo el uso de la misma. El hecho de que la información tenga un coste y, por lo tanto, los mercados financieros no sean totalmente eficientes, hace estratégica la inversión en esta tecnología para la producción y distribución de servicios financieros (Leland y Pyle 1997; Freixas y Rochet 1997). Ahora bien, la tecnología por sí misma no genera beneficios en términos de mayor transparencia para los usuarios y de menores costes para los intermediarios financieros. Para que estos beneficios se materialicen, ha sido necesario que se transformen los modelos de negocio de las entidades de depósito y las arquitecturas de los mercados financieros (Mishkin y Strahan 1999).

La tecnología ha revolucionado la actividad bancaria minorista, haciendo posible que las entidades puedan romper su cadena tradicional de creación de valor y permitiendo que la producción de servicios financieros y su distribución se conviertan en dos negocios distintos.

De esta forma, algunas de las principales características de los servicios bancarios han sido alteradas sustancialmente por la aplicación de nuevas tecnologías a la operativa de las empresas del sector. Así, características tradicionales como la intangibilidad de los servicios ofrecidos por las entidades bancarias y la obligación de mantener un contacto directo entre el proveedor del servicio y su cliente, han sido puestas en entredicho por los sistemas automatizados de banca a distancia puesto que la banca a distancia precisamente posibilita el suministro de tales servicios, sin contacto empleado-cliente, y la estandarización y distribución en masa permite que los servicios puedan ser transportados hasta el lugar en el que se localice el terminal de autoservicio. 
Estos cambios han traído consigo implicaciones profundas y de muy diversa índole sobre la estructura competitiva del sector (Banco Central Europeo 1999 y Bernal 2002):

- Las sucursales, a corto plazo seguirán siendo el eje central de la actividad bancaria; sin embargo, a largo plazo, por motivos de presión de la competencia, las entidades tendrán que sustituir sucursales bancarias por nuevos canales automatizados.

- Desde el punto de vista del personal que trabaja en la entidad, el cambio de naturaleza en la actividad bancaria no tiene porqué afectar necesariamente de manera negativa al empleo en el sector. La reducción de empleados en las sucursales se verá compensada por la incorporación de personal de marketing y ventas, y empleados técnicos cualificados para manejar las nuevas tecnologías.

- Se espera un aumento futuro de las actividades de outsourcing, especialmente en aquellas con elevado soporte tecnológico y en las no propiamente bancarias. Las nuevas tecnologías no darán lugar a la deslocalización de actividades.

- Las nuevas tecnologías no forzarán fusiones bancarias o adquisiciones, incluso en algunos casos pueden obstaculizarlas debido a la incompatibilidad de tecnologías entre las entidades. Respecto a las alianzas, las nuevas tecnologías de la información incrementarán la cooperación entre entidades para compartir gastos de desarrollo, alcanzar economías de escala, suministrar sistemas de pago inoperables, etc.

- Los canales de banca a distancia facilitarán la competencia de entidades ajenas al sector bancario, especialmente en actividades relacionadas con inversiones financieras, seguros, servicios de pago y de consulta.

- Las nuevas tecnologías permitirán al sector bancario aprovecharse aún más de la red comercial para ofertar sus productos financieros, utilizando los lugares físicos donde se ubican actualmente los supermercados.

- Reducción de costes unitarios como resultado del uso de sistemas automatizados. Ello será debido, entre otros aspectos, a la sustitución del trato personalizado por el de sistemas automatizados, a las economías de escala, a la racionalización de la producción, a la estandarización de procedimientos bancarios, a la mayor rapidez y mejora en el tratamiento de la información del consumidor, etc. 
- Existe incertidumbre acerca del efecto que las nuevas tecnologías tendrán sobre los gastos operativos generales. Su reducción sólo es posible bajo ciertas circunstancias (suficiente masa crítica de operaciones, eliminación de redundancias, no duplicar canales, etc.). En general, los especialistas creen que los costes totales únicamente se reducirán en el largo plazo.

- Las nuevas tecnologías tienen efectos contrarios sobre la lealtad del cliente. En primer lugar, permite recabar información sobre su comportamiento y gustos, lo que posibilita ofertar a los clientes productos específicos que cubran sus necesidades; en segundo lugar, facilitan al cliente el acceso a información de la competencia. Los especialistas aún no observan ninguna reducción en la lealtad de la clientela. Además, el uso de la banca a distancia reduce la importancia de las sucursales como canal de distribución y disminuye las barreras de entrada al sector.

- Existe poca diferenciación actual entre los precios de los servicios financieros a distancia y los de los canales tradicionales (excepto en algunos países en el caso de la banca por Internet). Se espera que el incremento de la competencia presione a la baja el precio de los servicios suministrados a través de canales con menores costes.

- Se espera un incremento de la rentabilidad en el medio y largo plazo debido al progreso tecnológico. A corto plazo, ello no será posible debido a los elevados gastos de establecimiento iniciales.

La mayoría de estas implicaciones se derivan de la desaparición de los límites geográficos y conceptuales de la industria bancaria. Y es que las nuevas tecnologías han conseguido trasladar la misma naturaleza del sector desde lo que tradicionalmente se había considerado estrictamente bancario hasta la industria de distribución de la información relacionada con los servicios financieros. Además, ha permitido difuminar los límites temporales (servicios de 24 horas, los 365 días del año) y geográficos (prácticamente en cualquier lugar del mundo desarrollado) en su canalización hacia los clientes.

Todo esto se ha visto traducido en una pérdida de importancia de la red de sucursales como canal de distribución y, en consecuencia, de su papel como barrera de entrada, facilitando así la intensificación de la competencia intersectorial e internacional en la oferta de servicios financieros. Al mismo tiempo, el factor humano ha tenido que adaptarse a la disminución de tareas repetitivas y de poco valor añadido (realizadas 
ahora por medios electrónicos, bien desde la propia entidad, o por otras distintas que trabajan en régimen de outsourcing) y al aumento de la importancia de otras relacionadas con el área de marketing.

Junto a todo lo anteriormente expuesto, la distribución a distancia de servicios financieros ha demostrado ser una importante fuente de ingresos para las entidades bancarias, especialmente vía comisiones, característica ésta muy apreciada en un contexto de continua reducción de márgenes financieros. Además, según Booz, Allen \& Hamilton (J.P. Morgan 2000: 18), la posibilidad de sustituir el tratamiento personalizado en las sucursales por uno estandarizado y ejecutado a distancia por medios electrónicos favorece la reducción de costes unitarios de distribución de servicios financieros, pudiendo llegar hasta el $99 \%$ en el caso del uso de Internet como canal de venta. No obstante, tal y como advierte el Banco Central Europeo (1999: 23-25), todo ello tendrá su reflejo en un incremento de la rentabilidad de la empresa bancaria sólo bajo determinadas circunstancias que rara vez se presentan en el corto plazo, como son, entre otras, la obtención de una masa crítica de clientes suficiente, la eliminación de redundancias en su operativa o de duplicidades de canales para atender a los clientes.

Tomando como ejemplo el caso de los cajeros automáticos, diversos autores (Revell 1983: 66; Chorafas 1989: 167; Humphrey 1994: 61 y 63; Maudos 1995: 11) han advertido que el reflejo de dicha reducción de costes unitarios en un ahorro de costes totales puede verse difuminado en el caso de que exista una sobre dotación de terminales o de que se produzca un aumento en el número de transacciones realizadas por parte de los clientes, debido a la disminución de los costes de transacción que conllevan estos sistemas. De hecho la relación positiva entre los costes totales medios de producción y la intensidad de uso de los cajeros automáticos en las entidades bancarias ha sido puesta de manifiesto, entre otros, por Humphrey (1994) y Berger (1985).

Esta realidad asociada a la banca a distancia en general, va a intensificarse de manera importante a medida que lo haga el uso de Internet para la venta de servicios bancarios. Entre los motivos que apoyan esta hipótesis se encuentra la extraordinaria dimensión del mercado asociado a Internet, formado por unos 31 millones de dominios registrados, de los que aproximadamente el 70 por cien son comerciales (NetNames International 2002); por unos 95 millones de ordenadores conectados a la red (OECD 2002), cada uno de los cuales se convierte en 
un punto de venta potencial, y por más de 500 millones de usuarios (Global Reach 2002; Nua Internet 2002).

Además, al tratarse de un sector que utiliza intensivamente la información y que rara vez precisa de la entrega física de sus productos o servicios, puede aprovecharse en gran medida de las ventajas asociadas a Internet como canal de venta, entre las que se encuentran la reducción de diversas fuentes de costes, la ampliación de mercados, la eliminación de barreras de entrada a otros mercados, el incremento del valor añadido de los productos ofertados, el suministro de información sobre el comportamiento de los clientes, etc. (OCDE 1998 y Sato y Hawkins 2001: 1).

Las implicaciones y consecuencias anteriormente referidas permiten definir un conjunto de debilidades y fortalezas para el sector bancario que brevemente resumimos en el siguiente cuadro:

\section{Análisis DAFO}

\begin{tabular}{|c|c|}
\hline Debilidades & Amenazas \\
\hline $\begin{array}{l}\text { - Los grandes bancos tienen que seguir redu- } \\
\text { ciendo costes para mantener su competitividad, } \\
\text { debido a sus grandes redes de oficinas. } \\
\text { - La rapidez se convierte en el factor principal } \\
\text { de éxito frente al tamaño. } \\
\text { - Fuertes inversiones en tecnología por parte de } \\
\text { los bancos de pequeño tamaño. } \\
\text { - Estructuras muy piramidales que dificultan dar } \\
\text { respuestas a la velocidad que exige Internet. } \\
\text { - Políticas de publicidad poco agresivas princi- } \\
\text { palmente en bancos pequeños y cajas de } \\
\text { ahorros. }\end{array}$ & $\begin{array}{l}\text { - Los bancos reducen el control sobre los } \\
\text { clientes, aumentando la rotación de éstos. } \\
\text { - Posible aparición de intermediarios que exigirán } \\
\text { una parte del margen del negocio. } \\
\text { - Gran desarrollo de los brokers online, los cuales } \\
\text { trabajan con unas comisiones significativamente } \\
\text { menores que la banca tradicional. } \\
\text { - Conflicto de canal con los canales tradicionales. } \\
\text { Posible aparición de nuevos entrantes } \\
\text { financieros y no financieros. }\end{array}$ \\
\hline Fortalezas & Oportunidades \\
\hline $\begin{array}{l}\text { - Los bancos disponen actualmente de una gran } \\
\text { base de clientes. } \\
\text { - El tradicional control que los bancos han } \\
\text { ejercido en el sector financiero les posiciona } \\
\text { favorablemente para aprovechar las oportuni- } \\
\text { dades del nuevo canal. } \\
\text { - Elevada capacidad tecnológica de los bancos } \\
\text { españoles. } \\
\text { - Fuertes marcas establecidas. } \\
\text { - Fuerte capacidad financiera para afrontar los } \\
\text { cambios. }\end{array}$ & $\begin{array}{l}\text { - Potencial para reducir costes operativos y } \\
\text { fijos. } \\
\text { - Nuevas formas de personalizar las relaciones } \\
\text { con los clientes y de entender sus necesidades. } \\
\text { - Posibilidad de incrementar la oferta de productos } \\
\text { y servicios aprovechando la clientela existente. } \\
\text { - Posibilidad de dirigirse a nuevos clientes. } \\
\text { - Se reducen las barreras para afrontar nuevos } \\
\text { negocios no bancarios. }\end{array}$ \\
\hline
\end{tabular}

Fuente: Andersen Consulting (2000). 
La apuesta por Internet, y las posibilidades abiertas al sector bancario han culminado en la definición y adopción de lo que algunos autores conocen como "nuevos modelos de negocio". La elección de un determinado modelo vendrá dado por el posicionamiento de la entidad en el negocio tradicional, la estrategia más o menos agresiva de la misma y sus posibilidades de inversión.

Entre los distintos nuevos modelos de negocio adoptados en los servicios online, destacan:

a) Sucursal Virtual: se trata de una filial cuya finalidad es comercializar productos y servicios propios o de terceros, conseguir clientes y ganancias en cuotas de mercado, en nuevos segmentos, territorios, con la menos canibalización posible de la clientela tradicional. Requiere precios muy atractivos y fuertes inversiones en publicidad y calidad de servicio.

b) Supermercado Financiero: es un modelo basado en la distribución de información y productos, centrados en un sector específico. Como agregadores de contenido, productos y servicios, el éxito de estos portales radicará en su capacidad de construir una fuerte relación con los usuarios, mediante la captación y posterior fidelización de los mismos.

c) One stop shopping: en un solo portal, los clientes deberían ser capaces de consultar la posición global de sus cuentas, acceder a todos los posibles productos, transferir fondos, negociar valores obtener información acerca de mercados, productos y empresas; recibir y pagar facturas, información personalizada, contratar hipotecas, tarjetas de crédito, seguros, etc.

d) Estrategia multicanal: la tendencia del mercado es que los clientes demanden cada vez más la posibilidad de tener acceso a todos los servicios financieros posibles en cualquier lugar, la presencia física de oficinas seguirá teniendo importancia a la hora de captar clientes. Las condiciones necesarias para el desarrollo de una estrategia de multicanal son:

- Contar con el respaldo adecuado de la alta dirección de la entidad y explicar internamente como un proyecto estratégico de la misma al servicio del conjunto organizacional.

- Asumir que Internet es un canal imprescindible para el negocio bancario, no una cuestión tecnológica. 
- Asumir que la tecnología no es hoy ni un problema ni un riesgo; de hecho, hasta cierto punto el retraso en este ámbito puede suponer ciertas ventajas.

\section{BIBLIOGRAFÍA}

AECE (2003, 2004) Estudio sobre Comercio Electrónico B2C.

AMIT, R. y ZoTT, C. (2001) "Value creation en e-business", Strategic Management Journal, 22, pp. 493-520.

ANDERSEN CONSULTING (2000) Esp@ña online. Ideas para afrontar la e-conomia. Investment Santander Central Hispano.

BAKOS, J.Y. (1991) "A strategic analysis of electronic marketplaces", MIS Quarterly, 15, 3, pp. 295-310.

- (1998) "The emerging role of Electronic Marketplaces on the Internet", Communications of the ACM, 48, 8, pp. 35-42.

- y BRYNJOLFSSON, E. (1993) "From vendors to partners: information technology and incomplete contracts in buyer-seller relationships", Journal of Organizational Computing, 3, 3, pp. 301-328.

BANCO Central EUROPEO (1999) The effects of technology on the EU banking systems. Francfort: European Central Bank.

BERGER, A. (1985) "The economics of electronic funds transfers", Board of Governors of the Federal Reserve System. Working Paper, October, tomado de Humphrey (1994).

BERNDT, E. y MORRISON, C. (1995) "High-tech capital formation and economic performance in U.S. manufacturing industries: an exploratory analysis", Journal of Econometrics, 65, pp. 9-43.

BRYNJOLFSSON, E. y HITT, L. (1996) "Paradox lost? Firm-level evidence of the returns to information systems spending", Management Science, 42, 4, pp. 541-558.

- y SMITH, M. (2000) "Frictionless commerce? A comparison of Internet and conventional retailers", Management Science, 46, 4, pp. 563-585.

- y YANG, S. (1996) "Information technology and productivity: a review of the literature", Advances in Computers, 43, pp. 179-214.

CHORAFÁS, D.N. (1989) High technology and the control of risk in banking. Londres: F.F./I.B.C. 
FREIXAS, X. y ROCHET, J.C. (1997) Microeconomics of Banking. Cambridge: MIT Press.

GlobAL ReSEARCH (2002) "Global Internet Statistics", www.glreach.com.

GonzÁLEZ, N. (coordinadora) (2003) "Las nuevas tecnologías de la información y la Comunicación: su impacto en el crecimiento económico", El Entorno Económico Actual. Madrid: Editorial Paraninfo.

GreENAN, N. \& MAIRESSE, J. (1996) "Computers and productivity in France: some evidence", National Bureau of Economics Research Working Paper, 5836.

GREENSTEIN, S. (2000) "Farming empirical research on the evolving structure of commercial Internet markets". E. BRYNJOLFSSON y B. KAHIN (eds.) Understanding the digital economy: data, tools and research. Cambridge: MIT Press.

GURBAXANI, V. y WHANG, S. (1991) "The impacts of information systems on organizations and markets", Communications of the ACM, 34, 1, pp. 54-73.

HaltiWAnger, J. y JaRmin, R.S. (2000) "Measuring the digital economy". E. BRYNJOLFSSON y B. KAHIN (eds.) Understanding the digital economy: data, tools and research. Cambridge: MIT Press.

HeSS, C.M. y KEMERER, C.F. (1994) "Computerized loan organization system: an industry case study of the electronic markets hypothesis", MIS Quarterly, 18, 3, pp. 251-274.

HUMPHREY, D.B. (1994) "Delivering deposit services: CA versus branches", Federal Reserve Bank of Richmond. Economic Quarterly, vol. 80, primavera, pp. 59-81.

JORGENSON, D. y STIROH, K. (1995) "Computers and growth", Economics of Innovation and New Technology, 3, Mayo, pp. 295-316.

LELAND, W. y PYLE, D. (1997) "Information asymetries, financial structure and financial intermediation", Journal of Finance, 32.

MAUDOS, J. (1995) "Technical change, cost and scale economies in the Spanish saving banks: the automatic teller machine", Research Papers in Banking and Finance $R P$ 95/13, Institute of European Finance, University of Wales, pp. 12-16.

MCAFEE, A. (2000a) "Economic impact of the Internet revolution: Manufacturing". Conferencia The E-business transformation: sector developments and policy implications. Universidad de California en Berkely. 
MISHKIN, F. y STRAHAN, P. (1999) What will technology do to financial structure?. Ed. by R. Litan \& A. Santomero, Brookings. Wharton Papers on Financial Services.

MORGAN, J.P. (2000) Online Finance Europe. Londres: J.P. Morgan Securities Ltd.

MORROW, K. y RÖEGUER, W.C. (2001) "Potential Output measurement methods, new economy influences and scenarios for 2001-2010: A comparison of the EU15 and the US", Dg ECFINC Economic Paper, 150.

NetNAmes INTERNATIONAL LTD. (2002) "World Domain Stats", www.domain stats.com.

NUA INTERNET (2002) "Nua Internet Surveys", www.nua.ie.

ORGANISATION FOR ECONOMIC CO-OPERATION AND DEVELOPMENT (OECD) (1998) "The economic and social impacts of electronic commerce: preliminary findings and research agenda", www.OECD.org/subject/e_commerce.

PORTER, M. (1980) Competitive Strategy. New York: Free Press.

- (2001) "Strategy and The Internet", Harvard Business Review, vol. 79, 3, pp. 63-78.

- y MILLAR, V.E. (1985) "How information gives you competitive advantage", Harvard Business Review, Julio-Agosto, pp. 149-160.

REVELL, J.R. (1993) Banking and electronic fund transfers. Paris: OCDE.

SATO, S. y HAWIKINS, J. (2001) "Electronic finance: an overview of the issues", Electronic Finance: A new Perspective and Challenges, Bank of International Settlements Papers, 7, Noviembre, pp. 1-12.

Solow, R. (1987) Review in New York Times Book Review, Julio 12, 1987. 